\title{
Reliability and Responsibility: A Theory of Endogenous Commitment
}

\author{
Matteo Triossi*
}

November 1, 2005

\begin{abstract}
A common assumption in Political Science literature is policy commitment: candidates maintain their electoral promises. We drop such assumption and we show that costless electoral campaign can be an effective way of transmitting information to voters. The result is robust to relevant equilibrium refinements. An unavoidable proportion of ambiguous politicians emerges, consistently with empirical findings.

JEL Classification: D72, P16, C73

Keywords: Information Transmission, Electoral Campaign, Endogenous Commitment.
\end{abstract}

\section{Introduction}

It is commonplace to say that electoral promises cannot be taken at face value. However parties and candidates invest a considerable amount of effort and resources in producing electoral messages. Presumably electoral campaign is believed to be a credible mean to attract voters' support. But if campaigns are a mere act of promising why should they influence citizens?

Intuitively campaigns convey information useful to predict future policies. So future policies should be predictable from present ones. Otherwise the electoral process could not accomplish its very objective of selecting and retaining politicians according to electors' views. Electoral campaigns, in order to be meaningful, must alter electors' beliefs about the policies the elected officials will implement. A widely employed explanation is that politicians and elected officials seek reelection. Electoral promises affect voters' expectations about the policies that will be chosen and provide a benchmark linking promises, policies and reelection (retrospective voting), because a credible threat to reelection

*Department of Economics. Universidad Carlos III. c/ Madrid 126, Getafe. 28903 Madrid, Spain, http://www.eco.uc3m.es/personal/info contacto/triossi.html email: mtriossi@eco.uc3m.es. I am especially grateful to Luis Corchón, Antonio Romero-Medina, Socorro Puy, Carlos Maravall, Leo Ferraris, Jordi Blanes i Vidal and Marc Möller for their useful comments. 
is imposed (see Barro (1973), Ferejohn (1986) and Austen-Smith and Banks (1989)).

But the disciplining role of electoral competition is only one face of the coin. Actually I will prove that electoral promises provide also a solution to the informational asymmetries between candidates and politicians. The difficulty arises because campaigns are cheap talk: when electoral messages alone are changed no agent's payoff differs.

Downs (1957) himself underlies the importance of the relationship between preelection statements and post-election behavior for rational voting being meaningful.

Now we try to prove that a party's ideology must be consistent with either (1) its actions in prior election periods, or (2) its statements in the preceding campaign (including its ideology), or (3) both... A party is reliable if its policy statements at the beginning of an election period-including those in its preëlection campaign-can be used to make accurate predictions of its behavior... A party is responsible if its policies in one period are consistent with its actions (or statements in the preceding period),.. (103-105)... The absence of reliability means that voters cannot predict the behavior of parties from what the parties say the will do. The absence of responsibility means party behavior cannot be predicted by consistently projecting what parties have done previously...We conclude that reliability is a logical necessity in any rational election system, and that responsibility-though not logically necessary-is strongly implied by rationality as we define it. Of course this conclusion does not prove that reliability and responsibility actually exist in our model. We can demonstrate that they do-and therefore that our model is rational-only by showing that political parties are inexorably driven by their own motives to be both reliable and responsible...(105-107). In our model it is necessary for each party's ideology to bear a consistent relation to its actions.... Any other procedure makes rational voting nearly impossible...(113)

But most of the classical models of electoral competitions like the HotellingDowns one assume that politicians are committed to their electoral engagements. The questions about the credibility of campaign promises are left unanswered.

Building on Downs' intuition the paper provides an explanation based both on informational asymmetries and dynamic aspects, in our case career concerns. Each one of the argument alone is not able to provide a satisfactory solution. The difficulty which arises is that campaigns are cheap talk: when electoral messages alone are changed no agent's payoff differs. Under complete information politicians cannot credibly commit to policies different than their favorite ones unless elections are infinitely repeated (Alesina (1988)). The result can be relaxed only allowing for indifference in voters' preferences (Aragonés et al 
$(2005))^{1}$. With the prospect of a unique election, costless electoral campaign cannot be meaningful (Harrington (1992a)) unless one drops the assumption of full policy enforceability (Harrington (1992b) $)^{2}$.

The paper which is closest to our approach is Harrington (1993a). He presents a model of finitely (twice) repeated elections under incomplete information under bilateral asymmetric information. Elected officials can choose between two policies. Candidates' and voters' types are the policies they think to be the most beneficial on income. The type space is finite and beliefs are not consistent with the common prior assumption. While voters' only care about their income, candidates' preferences are lexicographic: they first care about holding the office and then about the policy they implement. In this case each politician prefers to implement the policy she beliefs the most effective. The author proves that there exist equilibria in which each candidate truthfully announces and implements her favorite policy.

This paper presents a model in which candidates' care both about office and about the policy they would implement if elected. Politicians' and voters' preferences are private information. But differently from Harrington (1993b) the type space is continuous and beliefs are derived from a common prior. The distributions of agents' preferences is symmetric with respect to the origin. Candidates' compete for election by announcing a particular policy. The campaign announcement is totally costless. The winning candidate implements a policy and runs for reelection against a randomly chosen opponent. We focus on symmetric and monotonic equilibria in which more centrist politicians are elected with higher probabilities and implement more centrist policies. Monotonic equilibria permit to rule out very unlikely behaviors where extremists present themselves as centrist, while moderates make an extremist campaign, and have an intuitive appeal. Furthermore we show that in all non-monotonic equilibrium electoral campaign is meaningful. To refine out-of-equilibrium beliefs with regard to totally unexpected policies. we use a refinement introduced by Bernheim and Severinov (2003) (see also Kartik (2005)) The refinement, called monotonic D1 Criterion adapts to monotonic environments the D1 criterion (Cho and Kreps (1987)). We characterize the set of these equilibria for any degree of candidates' policy implication., where incumbents' motives are mainly reelection. Innovating on Harrington (1993a) we find that not only reelection pressures but also policy motivations can give relevance to electoral promises. Necessary conditions for informative campaign are a sufficiently high degree of policy concern on candidates' side and the ability of the electorate to credibly threaten the incumbent about her reelection perspectives. Candidates suffer the tension between pleasing their constituencies and seeking the reelection. The cost of ambiguity is to implement policies that are faraway from candidate's favorite one. So extremists are less willing to fully pay it. But they do pay a price, even when they

\footnotetext{
${ }^{1}$ The authors themselves admit that this approach does not seems a "compelling explanation because of how campaign promises can have effect since it rests on the existence of a nontrivial set of indifferent voters".

${ }^{2}$ Costly electoral campaign can be relevant (Banks (1990) and Callander and Wilkie $(2005))$.
} 
fully reveal their preferences. It is because they are forced to please the centrist electorate in order to enhance their election chances ${ }^{3}$. Centrist candidates prefer to be ambiguous in order to increase their election perspectives. They act as pure office-seekers. Reliability as commitment to the electoral promises of pert of the politicians emerges endogenously. In the same way responsibility appears, present policies can be useful proxies to predict future ones. Relaxing the refinement criterion other equilibria with relevant campaign emerge. But ambiguous, or dishonest behavior cannot be eliminated, independently on the equilibrium refinement used. There will be always politicians who act only to maximize their probability of reelection. Their share can only be reduced by an high degree of policy concern, which increases the costs of implementing a centrist policy. The ambiguity of centrist politicians captures a feature that Harrington (1993a) was not able to account for: the partial, even if relevant responsiveness of policies to electoral announcements found by empirical work (see Harrington (1992a) and (1992b)). The result also connects with the debate on the nature of political center. It is compatible with the vision of a political center lacking of a well defined ideology and better defined by its opportunistic behavior, which is quite popular between the general public (see Hazan (1997)).

The structure of the paper is the following. In section 2 we describe agents' behavior and we introduce the characteristics of electoral competition. and equilibrium. In Section 3 we present some preliminary results on electoral equilibrium that clarify our choices and we prove the impossibility of fully honest behavior. Section 4 introduces the equilibrium refinement that we will use in Section 5 to characterize a relevant set of equilibria. Finally Section 6 will draw the conclusion and possible directions of future research in the field. An Appendix contains the proofs of the results that are not included in the main text.

\section{The Model}

Candidates' and voters' preferences are private knowledge. Candidates compete for office by making campaign announcements. The winner chooses a policy taking in account reelection perspectives. There are two elections. The policy space is $P=[-D, D]$, where $D>0$. There are two candidates: $R$ (ight) and $L(\mathrm{eft})$. Candidates policy intentions (or types) are assumed to be independent and symmetric random variables. Candidate $R$ type, $\alpha_{R} \in[0, D]$ is drawn from the $\operatorname{cdf} F_{R}(\cdot)$ with continuos density, $f_{R}(\cdot)=F_{R}^{\prime}(\cdot)$. where $f_{R}(\alpha)>0$ if and only if $\alpha \in[0, D]$. Candidate $L$ 's policy intentions have symmetric density, $f_{L}\left(\alpha_{L}\right)=$ $f_{R}\left(-\alpha_{L}\right)$ for all $\alpha_{L} \in P_{L}=[-D, 0]$. Let $P_{R}=[0, D]$ and $P_{L}=[-D, 0]$ be candidate $R$ and candidate $L$ policy space, respectively.

Then $E_{R}(x)=\int_{0}^{D} \alpha f(\alpha) d \alpha=-\int_{-D}^{0} \alpha f(-\alpha) d \alpha=E_{L}(x)$ and $E_{R}\left(x^{2}\right)=$

\footnotetext{
${ }^{3}$ The symmetry assumptions on the distribution of preferences imply that the mass of voters is concentrated in the origin, in the case of an unimodal distribution.
} 
$\int_{0}^{D} \alpha^{2} f(\alpha) d \alpha=\int_{-0}^{0} \alpha^{2} f(-\alpha) d \alpha=E_{L}\left(x^{2}\right)$.

Set $E(x)=E_{R}(x)>0$ and set $E\left(x^{2}\right)=E_{R}\left(x^{2}\right)>0$.

In the campaign stage each candidate $j=L, R$ can send a message $m \in$ $P_{j}$. Based on campaign announcements $\left(m_{R}, m_{L}\right)$ each voter casts her vote for one of the candidate. We assume that there are $n$ electors, where $n \in \mathbf{N}$ is odd and publicly known. Once in office, the winning candidate implements a policy from her policy space, simultaneously a challenger is selected from the original distribution. Each voter observe incumbent's policy choice and, taking in account her policy announcement casts a vote to confirm or to fire her. Challenger's policy intentions are drawn from the original distribution $F_{L}{ }^{4}$.

A voter of type $v \in[-D, D]$ has preferences represented by the following utility function, $V(s, v)=-(\alpha-s)^{2}$.

At each election, independently across time, a median voter, $m_{v}$ is drawn from a symmetric distribution $G$ on $[-D, D]$ with continuos density, $g(\cdot)=G^{\prime}(\cdot)$. The assumption is equivalent to have a known median voter in 0 with a symmetric unknown idiosyncratic bias (see Austen-Smith and Banks (2005)).

Candidate $\alpha$ 's utility from winning the election is, at each period

$$
U(s, \alpha)=y-k(\alpha-s)^{2}
$$

where $s$ is the implemented policy and $y>0$ is the values she assigns to the office. $k>0$, measures candidate's degree of policy implication. A defeated candidate gets 0 utility $^{5}$

Let $\pi_{i}$ be the probability candidate $R$ wins the election $i$, for $i=1,2$. If an incumbent is confirmed there is not another election to present to so she will implement her favorite policy. From implementing policy $s_{R} \in P_{R}$ a candidate of type $\alpha \in[0, D]$ will derive utility

$$
U_{R}\left(\pi_{1}, \pi_{2}, s_{1}, s, \alpha\right)=\pi_{1}\left[y-k\left(\alpha-s_{R}\right)^{2}+\pi_{2} \delta y\right]
$$

where $\delta \in(0,1)$ is her intertemporal factor discount. At the first period candidate $R$ is free to make a policy announcement on $M \subset[0, D]$. Candidate $L$ 's. Preferences are defined symmetrically, with the same intertemporal factor discount, $\delta$ and candidate degree of policy implication.

\subsection{Voters' behavior}

Let $m_{v}$ be the median voter. Let $\mu$ be her beliefs about candidates' policy preferences She votes for candidate $R$ if and only if $E\left[\left(m_{v}-\alpha_{R}\right)^{2} \mid \mu\right]<$

\footnotetext{
${ }^{4}$ From Harrington (1992a and b) it follows that any campaign stage before the last election is irrelevant.

${ }^{5}$ The results can straightforwardly be generalized to the case in which candidates care also about the policy of their opponent.
} 
$E\left[\left(m_{v}-\alpha_{L}\right)^{2} \mid \mu\right]$ which is if and only if $m_{v}>e(\mu)$ where

$$
e(\mu)=\frac{1}{2} \frac{E\left[\alpha_{R}^{2} \mid \mu\right]-E\left[\alpha_{L}^{2} \mid \mu\right]}{E\left[\alpha_{R} \mid \mu\right]-E\left[\alpha_{L} \mid \mu\right]}
$$

is the decisive median voter.

Then $R$ is elected with probability $\pi(\mu)=1-G(e(\mu))=\frac{1}{2}+G(-e(\mu))$ given $G$ symmetry.

Remark 1 If a type $\alpha>0$ is matched against a challenger randomly drawn from $F_{L}$ her decisive median voter is

$$
e(\alpha, f(\cdot))=\frac{1}{2} \frac{\alpha^{2}-\int_{0}^{D} \beta^{2} f(\beta) d \beta}{\alpha+\int_{0}^{D} \beta f(\beta) d \beta}
$$

We will denote by $\pi((\alpha,, f(\cdot)))$ her probability of election. If types in $\left(\alpha_{1}, \alpha_{2}\right)$ pool together their decisive median voter is

$$
e\left(\left[\alpha_{1}, \alpha_{2}\right], f(\cdot)\right)=\frac{1}{2} \frac{\int_{\alpha_{1}}^{\alpha_{2}} \beta^{2} f(\beta) d \beta-\left(F\left(\alpha_{2}\right)-F\left(\alpha_{1}\right) \int_{0}^{D} \beta^{2} f(\beta) d \beta\right.}{\int_{\alpha_{1}}^{\alpha_{2}} \beta f(\beta) d \beta+\left(F\left(\alpha_{2}\right)-F\left(\alpha_{1}\right) \int_{0}^{D} \beta f(\beta) d \beta\right.}
$$

We will denote by $\pi\left(\left(\alpha_{1}, \alpha_{2},, f(\cdot)\right)\right)$ their probability of election. Using elementary Analysis it can be shown that $e\left(\alpha_{3}, f(\cdot)\right)>e\left(\left[\alpha_{1}, \alpha_{2}\right], f(\cdot)\right)>$ $e\left(\alpha_{0}, f(\cdot)\right)$ if $\alpha_{3}>\alpha_{2}>\alpha_{1}>\alpha_{0}>0 . \quad e(\alpha, f(\cdot))$ is strictly increasing in $\alpha . \quad e\left(\left[\alpha_{1}, \alpha_{2}\right], f(\cdot)\right)$ is strictly decreasing in $\alpha_{1}, \alpha_{2}$ (separately). Finally $\lim _{\alpha_{1} \rightarrow \alpha_{2}^{-}} e\left(\left[\alpha_{1}, \alpha_{2}\right], f(\cdot)\right)=e\left(\alpha_{2}, f(\cdot)\right)$ and $\lim _{\alpha_{2} \rightarrow \alpha_{1}^{+}} e\left(\left[\alpha_{1}, \alpha_{2}\right], f(\cdot)\right)=e\left(\alpha_{1}, f(\cdot)\right)$.

\section{$2.2 \quad$ Electoral Equilibrium}

A campaign strategy for candidate $j=R, L$ is a function $m_{j}: P_{j} \rightarrow P_{j}$. A campaign strategy is a "cheap talk" announcement of a policy by candidate $j$. If elected, candidate $j$ has to choose a policy $\sin P_{j}$. A policy strategy for incumbent $j$ is a function $s_{j}: P_{j}^{2} \times P_{k} \rightarrow P_{j}, j \neq k$.

A voting strategy is a 4-uple $\left(r_{1 j}, r_{2 j}\right)_{j=R, L}$ where $r_{1 j}: P_{R} \times P_{L} \times P \rightarrow$ $\left\{0, \frac{1}{2}, 1\right\}, r_{1 R}+r_{2 L}=1$ and $r_{2 j}: P_{R} \times P_{L} \times P_{j} \times P \rightarrow\left\{0, \frac{1}{2}, 1\right\} \cdot r_{1 j}\left(m_{R}, m_{L}, x\right)$ represents the probability the median voter votes for candidate $j$ at the first election, when she is of type $x$ and has observed electoral messages $\left(m_{R}, m_{L}\right)$. If $j$ results elected after campaign messages $\left(m_{R}, m_{L}\right) r_{2 j}\left(m_{R}, m_{L}, s_{j}, x\right)$ is the probability the median voter confirms her in the office when she implements policy $s_{j}$.

Remark 2 As each incumbent is opposed to a randomly chosen challenger there is no loss of generality in consider policy strategies independent from the other candidate campaign message, of the form $s_{j}: P_{j}^{2} \rightarrow P_{j}$. And there is no loss of generality in considering second stage election strategies independent on first stage loser's campaign, which is $r_{2 j}: P_{j}^{2} \times P_{j} \times P \rightarrow\left\{0, \frac{1}{2}, 1\right\}$. 
A belief at the first election about candidates is a function $\mu_{1}$ from the cartesian product of campaign messages $P_{L} \times P_{R}$ to the set of joint probability distributions on $P^{2}$. A belief at the second election is a function $\mu_{2}$ from the cartesian product of campaign messages, first stage voting outcomes, and policy outcomes to the set of joint probability distributions on $P^{2}$.

Definition 1 An electoral equilibrium consists of strategies $\left(m_{j}, s_{j}\right),\left(r_{1 j}, r_{2 j}\right)$ and beliefs $\left(\mu_{1}, \mu_{2}\right)$ such that for $j, k=R, L, j \neq k$

(1) $m_{j}(\alpha)$ maximizes in $m$

$$
\begin{aligned}
& \int_{P_{k}} \int_{[-D, D]} r_{1 j}\left(m, m_{k}(\beta), x\right)\left[y-k\left(\alpha-s_{j}(\alpha)^{2}\right] f(\beta) g(x) d \beta d x+\right. \\
& \int_{P_{k}} \int_{[-D, D]^{2}} r_{1 j}\left(m, m_{k}(\beta), x_{1}\right) r_{2 j}\left(m_{1}, s_{j}(\alpha), x_{2}\right) \delta y f(\beta) g\left(x_{1}\right) g\left(x_{2}\right) d \beta d x_{1} d x_{2}
\end{aligned}
$$

for all $\alpha \in P_{j}$

(2) $s_{j}(\alpha, m)$ maximizes in $s_{j} \in P_{j}$

$$
\left.-k\left(\alpha-s_{j}\right)^{2}+\int_{[-D, D]} r_{2 j}\left(m(\alpha), s_{j}, x\right)\right) \delta y g(x) d x
$$

for all $(\alpha, m) \in P_{j}^{2}$

(3) $r_{1 j}\left(m_{R}, m_{L}, x\right)=1$ if $E\left[\left(x-s_{j}(\cdot)\right)^{2} \mid m_{R}, m_{L}\right]<E\left[\left(x-s_{k}(\cdot)\right)^{2} \mid m_{R}, m_{L}\right]$ $r_{1}\left(m_{R}, m_{L}, x\right)=\frac{1}{2}$ if $E\left[\left(x-s_{j}(\cdot)\right)^{2} \mid m_{R}, m_{L}\right]=E\left[\left(x-s_{k}(\cdot)\right)^{2} \mid m_{R}, m_{L}\right]$ $r_{1}\left(m_{R}, m_{L}, x\right)=0$ if $E\left[\left(x-s_{j}(\cdot)\right)^{2} \mid m_{R}, m_{L}\right]>E\left[\left(x-s_{k}(\cdot)\right)^{2} \mid m_{R}, m_{L}\right]$ for all $\left(m_{R}, m_{L}, x\right) \in P_{R} \times P_{L} \times P_{L}$. Expectations are taken with respect to $\mu_{1}$

(4) $r_{2 j}(m, s, x)=1$ if $E\left[\left(x-\alpha_{j}\right)^{2} \mid m_{R}, s\right]<E\left[\left(x-\alpha_{k}\right)^{2}\right]$

$r_{2 j}(m, s, x)=\frac{1}{2}$ if $E\left[\left(x-\alpha_{j}\right)^{2} \mid m_{R}, s\right]=E\left[\left(x-\alpha_{k}\right)^{2}\right]$

$r_{2} j(m, s, x)=0$ if $E\left[\left(x-\alpha_{j}\right)^{2} \mid m_{R}, s\right]>E\left[\left(x-\alpha_{k}\right)^{2}\right]$

for all $(m, s, x) \in P_{j}^{2} \times P$. Expectations are taken with respect to $\mu_{2}$

(5) Expectations are computed using Bayes' rule whenever possible

Conditions (1) and (2) say that each candidate's electoral and policy strategies are sequentially optimal given her opponent's strategies and voters' decision. Conditions (3) and (4) say that voters' decisions are optimal at each election, given their beliefs.

Definition 2 An electoral equilibrium is symmetric whenever $\left(m_{R}(\alpha), s_{R}(\alpha)\right)=$ $-\left(m_{L}(-\alpha), s_{L}(-\alpha)\right)$ for all $\alpha \in[0, D]$ 


$$
\begin{aligned}
& \text { Set } \pi_{1 R}\left(m_{R}, m_{L}(\cdot)\right)=\int_{[-D, D]^{2}} \int_{P_{L}} r_{1}\left(m_{R}, m_{L}(\beta), x\right) f(\beta) g(x) d \beta d x \\
& \text { Set } \pi_{2 R}\left(m_{R}, s\right)=\int_{[-D, D]} \int_{P_{L}} r_{21}(m, s, x) g(x) d x
\end{aligned}
$$

and define analogous quantities for candidate $L . \pi_{1 R}$ and $\pi_{2 R}$ represent candidate $R^{\prime}$ probabilities of winning the first and the second election, respectively.

Let $\mu_{2 R}(\alpha, \cdot \mid m, s)$ be the marginal distribution of $R^{\prime}$ s type induced by message $m$ and policy $s$.

Candidate $R$ 's payoff is $\pi_{1 R}\left(m_{R}, m_{L}(\cdot)\right)\left(U(s, \alpha)+\pi_{2}\left(m_{R}, s\right) \delta y\right)$.

An electoral pool is a set $\Omega \subset[-D, D]$ such that, for some $m \in[-D, D]$, $m_{R}(\alpha)=m$ or $m_{L}(\alpha)=m$ for all $\alpha \in \Omega$.

A policy pool is a set $\Omega \subset[-D, D]$ such that, for some $s \in[-D, D], s_{R}(\alpha)=s$ or $s_{L}(\alpha)=s$ for all $\alpha \in \Omega$.

Definition 3 An electoral equilibrium is monotonic if

(1R) $\pi_{1 R}\left(m_{R}(\alpha), m_{L}(\cdot)\right)$ and $\pi_{2 R}\left(m_{R}(\alpha), s(\alpha)\right)$ are decreasing on $[0, D]$, and $s_{R}(m(\alpha), \alpha)$ is increasing on $[0, D]^{6}$

$(1 L) \pi_{1 R}\left(m_{R}(\cdot), m_{L}(\alpha)\right)$ and $\pi_{2 R}\left(m_{R}(\alpha), s(\alpha)\right)$ is increasing on $[-D, 0], s_{L}(m(\alpha), \alpha)$ is decreasing on $[-D, 0]$

In words an equilibrium is monotonic if more centrists candidates have higher probabilities of being elected, and implement more centrist policies. We impose monotonicity only on equilibrium messages. The following Remark points out the strict relation the connectivity properties. of monotone equilibria.

Remark 3 From the definition of monotonic equilibrium it follows that, at equilibrium:

(1) For all $m \in[0, D], \Omega(m)=\{\alpha: m(\alpha)=m\}$ is connected

(2) For all $m, s \in[0, D], \Omega(m, s)=\{\alpha: s(m, \alpha)=s\}$ is connected. In particular for each $\alpha \Omega(m(\alpha), s(\alpha))$ are connected.

(3) If $s(m(\alpha), \alpha)=s\left(m\left(\alpha^{\prime}\right), \alpha^{\prime}\right)$ then $\pi_{1}(\alpha)=\pi_{1 R}\left(\alpha^{\prime}\right)$ and $\pi_{2}(\alpha)=\pi_{2 R}\left(\alpha^{\prime}\right)$.

There is no loss of generality in assuming that candidates having the same probability of election at the first stage use the same electoral campaign (we assume candidates only use pure strategies) (3) can be written as (4) $s(m(\alpha), \alpha)=s\left(m\left(\alpha^{\prime}\right), \alpha^{\prime}\right) \Rightarrow m(\alpha)=m\left(\alpha^{\prime}\right)$.

Electoral and policy pools are connected in monotonic equilibria. So monotonicity helps to get rid of very unlikely equilibria. For instance situations in which centrists and moderates present different electoral platforms, but extremists pool with centrists. Furthermore monotonic equilibria have an intuitive appeal in our setup in which median voter's distribution is symmetric around the origin.

${ }^{6}$ For decreasing/increasing we mean weakly decreasing/increasing, otherwise we will say strictly decreasing/increasing. 
All along the paper we will devote our attention to symmetric monotonic equilibria. Then in the analysis it suffices to consider only one of the two candidates. We will analyze $R$ strategies omitting the subscript $R$, when there is no risk of ambiguity. At the same time we will use $s(\alpha)$ for $s(m(\alpha), \alpha), \pi_{1}(\alpha)$ for $\pi_{1}\left(m_{R}(\alpha), m_{L}(\cdot)\right)$ and $\pi_{2}(\alpha)$ for $\pi_{2}(m(\alpha), s(\alpha))$.

\section{Preliminary results on monotonic equilibria}

The first result provide an additional reason that makes of monotonic equilibrium a reasonable choice in this environment. Each electoral equilibrium is, locally, monotonic. In any electoral pool equilibrium policies are monotonic and second stage election probabilities are decreasing.

Lemma 1 Let $m \in P$ be a campaign message. Then in any symmetric equilibrium.

(i) $s(\alpha, m)$, is increasing on $\Omega(m)$.

(ii) $\pi_{2}(m, s(\alpha))$ is decreasing on $\Omega(m)^{7}$.

Symmetric claims hold for candidate $L$.

\section{It follows}

Corollary 1 In any non monotonic equilibrium the electoral campaign is meaningful.

The next result shows that, in any monotonic equilibrium, after a policy pool there will be a set of unused policies. We will make frequent use of it. In particular it implies that after a policy pool the policy function has a discontinuity.

Lemma 2 Let $s$ be equilibrium policy strategies and let $\alpha_{1}<\alpha_{2}$ and assume $s(\alpha)=s$ for all $s \in\left(\alpha_{1}, \alpha_{2}\right)$ then there exists $h>0$ such that policies in $(s, s+h)$ are not used.

In any equilibrium some candidate lies and imitates the policy implemented by a more centrist politician in order to increase her probability of winning the elections. As Corollary it follows that no separating equilibrium exists

Lemma 3 In a monotonic equilibrium, if $s(\alpha)$ is separating in $\left[\alpha_{1}, \alpha_{2}\right)$ then $s(\alpha) \neq \alpha$ on $\left[\alpha_{1}, \alpha_{2}\right)$. More $s(\alpha)<\alpha$ on $\left[\alpha_{1}, \alpha_{2}\right)$.

Proposition 4 There is no policy separating monotonic equilibrium. Then there is no full separating equilibrium.

\footnotetext{
${ }^{7}$ Property (i) holds in any electoral equilibrium, either symmetric or asymmetric as follows from the proof of the result.
} 
Proof. Otherwise, from Lemma $3 s(0)>0$. But then type $s(0)>0$ could profitably deviate by imitating 0 , because it would increase her election probabilities and would save her policy costs, a contradiction. Any full separating equilibrium is equivalent to a monotonic equilibrium so the second claim follow from the first.

\section{The MD1 refinement}

In this section we present an equilibrium refinement, introduced by Bernheim and Severinov (2003) and studied also in Kartik (2005) for cheap talk extensions of one round signalling games. Differently from Kartik (2005) in our model there are two senders and receiver's type is unknown. Furthermore cheap talk and costly signalling are not simultaneous. We then adapt the refinement to our framework. The refinement is applied only to policies that are never used in equilibrium.

Before the MD1 criterion is defined we present another piece of notation. We will refer to the lower and highest probability of election, following a given policy $s$. For all $s \in[0, D]$ set

$$
\begin{aligned}
& \pi_{l R}(s)=\sup _{s_{R}(\alpha)>s} \pi_{2 R}(\alpha), \text { if } s_{R}(\alpha)>s \text { for some } \alpha \in[0, D] \\
& \pi_{l R}(s)=\pi_{2 R}(D, f(\cdot)) \text { otherwise }
\end{aligned}
$$

and

$$
\begin{aligned}
& \pi_{h R}(s)=\inf _{s_{R}(\alpha)<s} \pi_{2 R}(\alpha), \text { if } s_{R}(\alpha)<s \text { for some } \alpha \in[0, D] \\
& \pi_{h R}(s)=\pi(0, f(\cdot)) \text { otherwise }
\end{aligned}
$$

Analogous quantities are defined for candidate $L$.

Definition 4 An electoral equilibrium satisfies the monotonic D1 (MD1) criterion if

(1) It is monotonic

(2) For all $m=\left(m_{R}\left(\beta_{R}\right), m_{L}\left(\beta_{L}\right)\right)$ and all $s \in[0, D]$ with $\mu\left(s \mid \beta_{R}, \beta_{L}\right)=0$ for all $\left(\beta_{R}, \beta_{L}\right) \in P_{R} \times P_{L}$. If there exists a non-empty set of types $\Omega \subset[0, D]$ such that, for each $\alpha \notin \Omega$, if there exists some $\alpha^{\prime} \in \Omega$ such that for all $\pi \in$ $\left[\pi_{l R}(s), \pi_{h R}(s)\right]$

$$
\pi_{1 R}(\beta)\left(y-k(s-\alpha)^{2}+\pi \delta y\right) \geq \pi_{1 R}(\alpha)\left(y-k\left(s_{R}(\alpha)-\alpha\right)^{2}+\pi_{2}(\alpha) \delta y\right)
$$

$$
\pi_{1 R}(\beta)\left(\left(y-k\left(s-\alpha^{\prime}\right)^{2}+\pi \delta y\right)>\pi_{1 R}\left(\alpha^{\prime}\right)\left(y-k\left(s_{R}\left(\alpha^{\prime}\right)-\alpha^{\prime}\right)^{2}+\pi_{2}\left(\alpha^{\prime}\right) \delta y\right)\right.
$$

(3) $\Omega$ is minimal with respect this property and $\mu(\cdot, \cdot \mid m, s)=\mu_{R}(\cdot) f(\cdot)$, where $\left.\operatorname{supp}_{R}(\cdot \mid m, s)\right) \subset \Omega$.

Analogous requirement is imposed for candidate $L$. 
In the case in which $\left[\pi_{l R}(s), \pi_{h R}(s)\right]$ is substituted by $[\pi(D, f(\cdot)), \pi(0, f(\cdot))]$ we would have an adaptation to our setup of the D1 criterion introduced by Cho and Kreps (1987). (2) extends the monotonicity requirements to out of equilibrium beliefs. If an elected official implements out of equilibrium policy $s$, she should expect of being reelected with probability between $\pi_{l R}(s)$ and $\pi_{h R}(s)$. The refinement assign positive probability only to those types who benefit most from this deviation.

\section{$5 \quad$ Equilibrium characterization and existence}

If $s(\cdot)$ is increasing then it has at most a countable set of discontinuity points and it is differentiable almost everywhere (see Royden (1988)). There is no loss of generality in assuming that electoral campaign is monotonic increasing and that $m(\alpha)=\alpha$ when type $\alpha$ is separating, and that agents having the same probability of being elected at the first election make the same announcement.

Let $\alpha_{1}<\alpha_{2}<\ldots<\alpha_{k}<\ldots$ be $s$ 's discontinuity points of $s_{R}$.

Lemma 4 If, at equilibrium types in $\left(\alpha_{i}, \alpha_{i+1}\right)$ are pooling on the same policy then they sent the same campaign message ${ }^{8}$.

Proof. Let $0 \leq \alpha<\alpha^{\prime}<\alpha^{\prime \prime}$. By contradiction assume some types in $\left(\alpha, \alpha^{\prime}\right) \subset$ $\left(\alpha_{i}, \alpha_{i+1}\right)$ sending message $m$ and let types in $\left(\alpha^{\prime}, \alpha^{\prime \prime}\right) \subset\left(\alpha_{i}, \alpha_{i+1}\right)$ sending message $m^{\prime}$ Let $\alpha^{\prime}+\varepsilon$ imitate type $\alpha^{\prime}-\varepsilon$. The gain in the probability of being elected at the first stage is bounded below by a strictly positive constant.The gain in second election probability is non negative. For $\varepsilon \rightarrow 0$ the loss in policy term goes to 0 by continuity so the deviation would be profitable for $\varepsilon$ small enough.

Observe that $\left.\alpha=\arg \max _{\alpha^{\prime}} \pi_{1}\left(\alpha^{\prime}\right)\right)\left[y-k\left(s\left(\alpha^{\prime}\right)-\alpha\right)^{2}+\pi_{2}\left(\alpha^{\prime}\right) \delta y\right]$

So almost everywhere.

$\left.\left.\pi_{1}^{\prime}(\alpha)\left[y-k(s(\alpha)-\alpha)^{2}+\pi_{2}(\alpha) \delta y\right]+\pi_{1}(\alpha)\right)\left[-2 k s^{\prime}(\alpha)\right)(s(\alpha)-\alpha)+\pi_{2}^{\prime}(\alpha) \delta y\right]=0$

If agents in $\left(\alpha^{\prime}, \alpha^{\prime \prime}\right)$ pool on the same campaign and on the same policy the inequality is $0=0$.

In particular, if all agents in $\left(\alpha^{\prime}, \alpha^{\prime \prime}\right)$ pool on the same campaign but use different policies $\left.\left(\pi_{1}(\alpha)\right)\right)^{\prime}=0$ so that

$$
\left.\left[-2 k s^{\prime}(\alpha)\right)(s(\alpha)-\alpha)+\pi_{2}^{\prime}(\alpha) \delta y\right]=0 \text { on }\left(\alpha^{\prime}, \alpha^{\prime \prime}\right)
$$

Remark 5 If $\pi_{1}$ and $\pi_{2}$ are $C^{1}$ and strictly decreasing both problems defined by the differential equations above and the terminal condition $S(D)=D$ have a unique solution such that $s(\alpha)<\alpha$ on $(D-\varepsilon, D)$ for arbitrary small $\varepsilon$. The

${ }^{8}$ Under the convention introduced in Remark 3 
result follows from Lemma 5 in the Appendix (see also Kartik (2005). Such solution is such that $s(\alpha)<\alpha$ on $(0, D)$. Furthermore $s(0)<0$, because if $s$ crossed the diagonal $s^{\prime} \rightarrow \infty$. This is impossible: if the graph cross the diagonal it must be from below because $s(\alpha)<\alpha$ on $(0, D)$.

Set for all $\alpha, \alpha^{\prime}$ such that, $m(\alpha)=m\left(\alpha^{\prime}\right)$ and all $\pi$, set

$$
T\left(\alpha, \beta, s, \pi_{2}\right)=\pi_{1}(\beta)\left(y-k(s-\alpha)^{2}+\pi_{2} \delta y\right)-\pi_{1}(\alpha)\left(y-k(s(\alpha)-\alpha)^{2}+\pi_{2}(\alpha) \delta y\right)
$$

Remark 6 Condition (2) can be written in this case as For all $m=\left(m_{R}\left(\beta_{R}\right), m_{L}\left(\beta_{L}\right)\right)$ and all $s \in[0, D]$ with $\mu\left(s \mid \beta_{R}, \beta_{L}\right)=0$ for all $\left(\beta_{R}, \beta_{L}\right) \in P_{R} \times P_{L}$. If there exists a non-empty set of types $\Omega \subset[0, D]$ such that, for each $\alpha \notin \Omega$, if there exists some $\alpha^{\prime} \in \Omega$ such that for all $\pi \in\left[\pi_{l}(s), \pi_{h}(s)\right]$

$$
T\left(\alpha, \beta, s, \pi_{2}\right) \geq 0 \Longrightarrow T\left(\alpha^{\prime}, \beta, s, \pi_{2}\right)>0
$$

$\Omega$ is minimal with respect this property and $\mu(\cdot, \cdot \mid m, s)=\mu_{R}(\cdot) f(\cdot)$, where $\left.\operatorname{supp}_{R}(\cdot \mid m, s)\right) \subset \Omega$.

When there is no risk of ambiguity we will write about $\beta, s, \pi_{2}$ we will write $T(\alpha)$ for $T\left(\alpha, \beta, s, \pi_{2}\right)$ and $T^{\prime}(\alpha)$ for $T_{1}\left(\alpha, \beta, s, \pi_{2}\right)$.

From Equation 1 it follows that

$$
T^{\prime}(\alpha)=2 k\left[\pi_{1}(\alpha)(\alpha-s(\alpha))-\pi_{1}(\beta)(\alpha-s)\right]
$$

Any monotonic D1 equilibrium is characterized by a cutoff type that divides pooling from separating types.

Proposition 7 Any symmetric monotonic D1 is essentially equivalent to an equilibrium in which, for all $i$ there exists $\alpha^{*} \in(0, D]$ such that

(1) $s_{R}(\alpha)=0$ on $\left[0, \alpha^{*}\right]$

(2) If $\alpha^{*}<D$ then $s_{R}(\alpha)$ is separating on $\left(\alpha^{*}, D\right]$ and $s_{R}(D)=D$.

Actually we can go further and characterize fully all monotonic D1 equilibria. We can divide the them in four categories:

(i) fully pooling equilibria in which all types pool in campaign and policy

(ii) campaign irrelevant but policy significative equilibria, in which all types send the same electoral message, but the more extremist types separate in policy

(iii) weakly expressive campaign equilibria in which centrists and extremists form different campaign pools but extremists separate in policy

(iv) expressive campaign equilibria where centrists pool on the same electoral promise and on the same policy and extremists separate both in campaign and in policy

The larger is $k$, the larger are the possibilities of relevant electoral campaign. The expressive campaign equilibria asymptotically converge to a fully separating equilibrium, in which electoral promises are maintained. 
Theorem 8 There exist $k_{0}<k_{1}<k_{2}$ and strictly decreasing functions $\alpha_{1}(k), \alpha_{2}(k), \alpha_{3}(k)$ with $\lim _{k \rightarrow \infty} \alpha_{i}(k)=0$ for $i=1,2,3$, such that

(i) If $k \leq k_{0}$ there are only fully pooling equilibrium in which $m(\alpha)=m(0)$

and $s(\alpha)=0$ for all $\alpha \in[0, D]$. If $k>k_{0}$ such an equilibrium is not MD1

(ii) For $k_{0} \leq k$ there exists a campaign irrelevant but policy significative $M D 1$ equilibria such that $m(\alpha)=m(0)$ for all for all $\alpha \in[0, D], s(\alpha)=0$ $\alpha \in\left[0, \alpha_{1}(k)\right], s(\alpha)$ is separating on $(\alpha(k), D]$

(iii) $k_{1} \leq k$ there exists a weakly expressive campaign equilibrium in which $m(\alpha)=m(0)$ for all $\alpha \in\left[0, \alpha_{2}(k)\right]$ and $m(\alpha)=m^{\prime}$ for $\alpha \in\left[\alpha_{2}(k), D\right] . s(\alpha)=0$ for $\alpha \in\left[0, \alpha_{2}(k)\right]$ and $s(\alpha)$ is separating on $\left(\alpha_{2}(k), D\right]$.

(iv) $k_{2} \leq k$ there exists a expressive campaign equilibrium in which $m(\alpha)=$ $m(0)$ for all $\alpha \in\left[0, \alpha_{3}(k)\right]$ and $m(\alpha)=\alpha$ for $\alpha \in\left[\alpha_{3}(k), D\right] . s(\alpha)=0$ for $\alpha \in\left[0, \alpha_{2}(k)\right]$ and $s(\alpha)$ is separating on $\left(\alpha_{3}(k), D\right]$.

Any symmetric MD1 equilibrium is essentially equivalent with one of the equilibria described above.

The existence part of Theorem 8 relies on the possibilities of threatening the incumbent of not confirming her in office if she shirks. It requires beliefs to be correlated outside of the equilibrium path ${ }^{9}$. It is like electors, when deceptioned by elected officials had doubt on optimality of their past actions and were seriously tempted to give rationally the support to the challenger. If they was wrong about one candidate why should not they be wrong about the other one, too?

In any case in the real world electoral disappointment does have an effect on electors. The model we presented does not capture this aspect because the idiosyncratic shocks defining median voter exact position is independent across periods and uncorrelated to actions. But electoral disappointment can be introduced as a shift of voters distribution, correlated with the degree of electoral fulfillment. To make things simple as possible assume that median voter distribution is shifted to left in the case of an $R$ incumbent, or to the right in the case of an $L$ incumbent of a fix factor $x>0$, if the elected officer deviates from the expected policy (ies $)^{10}$. The reader can easily verify that the claim of Theorem 8 holds even if we impose electors' beliefs about the two candidates to be independent. More precisely, if $x \geq D-\frac{D^{2}-\int_{0}^{D} \beta f(\beta) d \beta}{D+\int_{0}^{D} \beta f(\beta) d \beta}$ the $k_{0}, k_{1}, k_{2}$ found in the proof of the result would stay the same ${ }^{11}$. Otherwise their value would be larger as it would harder to induce extremists not to pool in campaign We conjecture that a similar result can be obtained also through a shock which is continuously dependent from the distance between expected policy and implemented one.

The MD1 refinement applies only to zero probabilities policies . It is strong

\footnotetext{
${ }^{9}$ Which is allowed by our definition of equilibrium.

${ }^{10}$ Excluding the case of a totally out of equilibrium policy, which defines the MD1 criterion.

$11 \frac{D^{2}-\int_{0}^{D} \beta f(\beta) d \beta}{D+\int_{0}^{D} \beta f(\beta) d \beta}$ is the minimal displacement that makes the median never voting $D$ against a challenger randomly drawn from $F_{L}$,
} 
enough to shrink down dramatically the set of possible equilibria. The key, as for Universal Divinity, is that we ask the support of the distribution to be minimal. If the function $T$ has a a unique maximizer, $\alpha$, then to such maximizer must be given probability one. Like in Banks (1990), this leads to equilibria characterized by a unique cutoff type. Dropping the minimality requirement would allow for a very large set of electoral equilibria even with expressive electoral promises. Consider the following case in which types in $\left(\alpha_{i}, \alpha_{i+1}\right)$ are pooling on policy $s$ (or they are separating) and the jump of the policy function is $h$. If the minimality requirement is dropped this is compatible with the support of median voter's beliefs being in whatever set containing $\alpha_{i+1}$. It is then compatible with equilibria in which a centrist and an extremist pool exist while the other types separate in campaign and in policy.

The claim of Proposition 4 relies on the boundedness of the type space. Allowing for an unbounded type space can lead to full separation in senderreceiver games with both costly messages and cheap talk (see Kartik (2005)). It is not the case here. We would of course obtain full separation in policy, but total pooling in campaign. The reason is that, asymptotically, candidates utilities is null, so it is the effect of career concerns. Very extremist candidates would be incomparably better off by maximizing their first election probability. Full separation could probably be obtained in the case in which candidates care also about challengers' implemented policy.

\section{Conclusions}

We have presented a model of electoral competition under incomplete information in which candidates care both about office and the policy. Introducing incomplete information and the dynamic aspects of a double election we have proved that electoral campaign is able to convey relevant information to voters even if it is not costly. In this direction we open a possibility for endogenous commitment of policy innovating on Harrington (1992a). The result is driven both on candidates' career concern and then the threat of failed reelection and the impossibility for candidates to sustain policies that are too faraway from their ideal ones. Extending Harrington (1993a) we find that not only reelection pressure but also policy motivation can give relevance to electoral promises. Despite of it we also proved that centrist electoral opportunism cannot be totally eliminated, but only reduced if candidates' degree of policy implication is high enough This is consistent with the empirical literature which estimates that only a part (even if relevant) of policies are responsive to electoral compromises.

The investigation can be extended in different directions. On the one hand toward the study of more complex models of competition. In our model the "world ends" after the second election. So just before it there is no place for meaningful electoral competition. Allowing for repeated interactions should make it relevant. An appropriate and realistic simple model would be the one of an overlapping generation of politicians that can stay in the office for a fixed number of terms. In this case challengers' campaign would be relevant. The 
threat to reelection imposed on the incumbent would be reinforced, and so the degree of commitment.

A partially unexplored field is about the nature itself of electoral campaign. It is usually modeled as a one-shot policy announcement (either costly or cheap). Despite of it electoral campaign are, in the real world repeated and longer interactions between electors and politicians. A lot of announcements are made, a lot of resources are devoted to test electors intentions and tastes through pools (see for instance Alvarez (1998)). It is probably the case that this matters a lot in the process of information transmission in two directions: parties both try to produce reliable information and at the same time the try to get information about electors. Actually it has been shown (see Krishna and Morgan (2004)) that repeated cheap talk iteration (conversation) extends the set of equilibria of the Crawford-Sobel model.

\section{References}

[1] Alesina, A. (1988) "Credibility and Policy Convergence in a Two-party System with Rational Voters" American Economic Review, 78:796-806.

[2] Alvarez, M. R. (1998) "Information and Elections", Revised Edition, Ann Harbor: University of Michigan Press.

[3] Aragonès, E., Palfrey, T. R. , and A. Postlewaite (2005) "Reputation and Rhetoric in Elections", PIER Working Papers, 05-021

[4] Austen-Smith, D. and J. Banks (1989) "Electoral Accountability and Incumbency" in Models of Strategic Choice in Politics, P. Ordeshook (ed.) Ann Harbor: University of Michigan Press.

[5] Austen-Smith, D. and J. Banks (2005) "Positive Political Theory II: Strategy and Structure" Ann Harbor: University of Michigan Press.

[6] Banks, J. and J. Sobel (1987) "Equilibrium selection in Signalling Games" Econometrica, 55:647-661

[7] Banks, J. (1990) "A Model of Electoral Competition with Incomplete Information", Journal of Economic Theory 50:309-325.

[8] Barro, R. (1973) "The control of Politicians: An Economic Model", Public Choice, 14:19-42.

[9] Bernheim, B. D. and S. Severinov (2003) "Bequests as Signals: An Explanation for the Equal division Puzzle", Journal of Political Economy 111:733-764.

[10] Callander, S. and S. Wilkie (2005) "Lies, Damned Lies and Political Campaigns", Working Paper. 
[11] Cho, I-K and D. Kreps "Signalling Games and Stable Equilibria" Quarterly Journal of Economics, 102:179-221

[12] Crawford, V. and J. Sobel (1982) "Strategic Information Transmission" Econometrica 50:1431-145.1

[13] Cukierman, A. and M. Tommasi (1998) "When Does It Take a Nixon to Go to China?" American Economic Review, 88:180-197.

[14] Downs, A (1957) "An Economic Theory of Democracy", New York: Harper and Row.

[15] Ferejohn, J. (1986) "Incumbent Performance and Electoral Control", Public Choice, 50:5-25.

[16] Fishel, J. "Presidents \& Promises", CQ Press, Washington DC

[17] Harrington, J.E (1992a) "Modelling the Role of Information in Elections", Mathematical Computer Modelling, 16:133-145.

[18] Harrington, J.E (1992b) "The Revelation of Information through the Electoral Process: An Exploratory Analysis" Economics an Politics, 4:255-275.

[19] Harrington, J.E (1993a) "The Impact of Reelection Pressures on the Fulfillment of Campaign Promises" Games and Economic Behavior, 5:71-97.

[20] Harrington, J.E (1993b). "Economic Policy, Economic Performance, and Elections", American Economic Review, 83:27-42.

[21] Hazan, R., Y. (1997) "Centre Parties. Polarization and Competition in European Democracies", Continuum, London and New York.

[22] Kartik, N. (2005) "Information Transmission with Cheap and AlmostCheap Talk" Working Paper.

[23] Krishna V. and J. Morgan (2004) "The art of conversation: eliciting information from experts through multi-stage communication", Journal of Economic Theory 117:147-179

[24] Matthews, S. A., M. Okuno-Fujiwara and M. Postlewaite (1991) "Refining Cheap-Talk Equilibria", Journal of Economic Theory, 55,247-273

[25] Pomper, G. M. "Elections in America", $2^{\text {nd }}$ Edition, Longman, New York

[26] Pontiriaguine L., (1969) "Equatións Différentielles Ordinaires", Editions Mir, Moscou

[27] Royden, H. L. (1988) "Real Analysis, Third Edition", Prentice Hall. 


\section{Appendix}

\subsection{Section 3}

Proof of Lemma 1 Let $0 \leq \alpha<\alpha^{\prime}$. Set $s=s(\alpha, m), s^{\prime}=s\left(\alpha^{\prime}, m\right), \pi_{2}=$ $\pi_{2}(m, s(\alpha, m))$ and $\pi_{2}^{\prime}=\pi_{2}^{\prime}\left(m, s_{1}\left(\alpha^{\prime}, m\right)\right.$.

(i) By contradiction let $s^{\prime}<s$. From incentive compatibility it follows

$$
\begin{aligned}
-k(s-\alpha)^{2}+\pi_{2} \delta y & \geq-k\left(s^{\prime}-\alpha\right)^{2}+\pi_{2}^{\prime} \delta y \\
-k\left(s-\alpha^{\prime}\right)^{2}+\pi_{2}^{\prime} \delta y & \geq-k\left(s-\alpha^{\prime}\right)^{2}+\pi_{2} \delta y
\end{aligned}
$$

Which is

$$
\begin{aligned}
\left(\pi_{2}-\pi_{2}^{\prime}\right) \delta y+k\left[\left(s^{\prime}-\alpha\right)^{2}-(s-\alpha)^{2}\right] & \geq 0 \\
\left(\pi_{2}^{\prime}-\pi_{2}\right) \delta y+k\left[\left(s-\alpha^{\prime}\right)^{2}-\left(s^{\prime}-\alpha^{\prime}\right)^{\prime 2}\right] & \geq 0
\end{aligned}
$$

Summing up the two inequalities

$$
\left(s^{\prime}-\alpha\right)^{2}-(s-\alpha)^{2}+\left(s-\alpha^{\prime}\right)^{2}-\left(s^{\prime}-\alpha^{\prime}\right) \geq 0
$$

With elementary algebra

$$
\left(\alpha-\alpha^{\prime}\right)\left(s-s^{\prime}\right) \geq 0
$$

The claim follows as $\alpha<\alpha^{\prime}$.

(ii) By contradiction let $\pi_{2}>\pi_{2}^{\prime}$ Assume that it is not the case. From (i) and the definition of monotonic equilibrium it follows that it cannot be the case that $\alpha$ and $\alpha^{\prime}$ separate or that $\alpha$ and $\alpha^{\prime}$ pool or that $\alpha^{\prime}$ pools with some other type and $\alpha$ separate. It must be the case that $\alpha$ pools and $\alpha^{\prime}$ separates. But then from Remark Connectivity the pool $\alpha$ belongs to is an interval $\left(\alpha_{1}, \alpha_{2}\right)$. In such a case the decisive median voter of $\alpha$ is $e(\alpha)=\frac{1}{2} \frac{\int_{\alpha_{1}}^{\alpha_{2}} \beta^{2} f(\beta) d \beta-\left(F\left(\alpha_{2}\right)-F\left(\alpha_{1}\right)\right) \int_{0}^{D} \beta^{2} f(\beta) d \beta}{\int_{\alpha_{1}}^{\alpha_{2}} \beta f(\beta) d \beta+\left(F\left(\alpha_{2}\right)-F\left(\alpha_{1}\right)\right) \int_{0}^{D} \beta f(\beta) d \beta}$ while the decisive median voter of $\alpha^{\prime}>\alpha$ is $e\left(\alpha^{\prime}\right)=\frac{1}{2} \frac{\left(\alpha^{\prime}\right)^{2}-\int_{0}^{D} \beta^{2} f(\beta) d \beta}{\alpha^{\prime}+\int_{0}^{D} \beta f(\beta) d \beta}>e(\alpha)$, a contradiction.

Proof of Lemma 2 From Remark $3\left(3^{\prime}\right) m(\alpha)=m$ on $\left(\alpha_{1}, \alpha_{2}\right)$. There is no loss of generality in assuming that $\left(\alpha_{1}, \alpha_{2}\right) \subset \Omega(m, s) \subset\left[\alpha_{1}, \alpha_{2}\right]$. We will write $s$ From monotonicity $s\left(\alpha_{2}\right) \geq s$.

Consider first the case $s\left(\alpha_{2}\right)>s$. Then policies in $\left(s, s\left(\alpha_{2}\right)\right)$ are not used in equilibrium.

Now let $s\left(\alpha_{2}\right)=s$ and set $\widehat{s}=\lim \alpha_{\alpha} \alpha_{2} s(\alpha)=\inf { }_{\alpha>\alpha_{2}} s(\alpha)$. Observe that $s(\alpha)>s$ for $\alpha>\alpha_{2}$. By contradiction let $\widehat{s}=s$. Set $\pi_{1 \varepsilon}=\pi_{1}\left(\alpha_{2}+\varepsilon\right)$, $\left.\pi_{1}=\pi_{10}, \pi_{2 \varepsilon}=\pi_{2 R} \alpha_{2}+\varepsilon\right), \pi_{2}=\pi_{20}$. It must be the case that $\pi_{2 \varepsilon}<\pi_{2}$ and $0<\pi_{1 \varepsilon} \leq \pi_{1}$ for all $\varepsilon>0$. From Remark $\pi_{2}-\pi_{2 \varepsilon}$ is bounded below by some positive constant $c$. Furthermore $\left(s\left(\alpha_{2}+\varepsilon\right)-\left(\alpha_{2}+\varepsilon\right)\right)^{2}>$ $\left(s-\alpha_{2}-\varepsilon\right)^{2}$ for all $\varepsilon>0$ Otherwise $\alpha_{2}+\varepsilon$ could profitably deviate by mimicking $\alpha_{2}$. 
$L(\varepsilon)$ is the net loss or the net gain to type $\alpha_{2}+\varepsilon$ from imitating type $\alpha_{2}$. As it is an equilibrium $L(\varepsilon) \leq 0$ for all $\varepsilon>0$.

For all $0<\varepsilon<\varepsilon^{*}$ set

$L(\varepsilon)=\pi_{1}\left(y-k\left(s-\alpha_{2}-\varepsilon\right)^{2}+\pi_{2} \delta y\right)-$

$\pi_{1 \varepsilon}\left\{y-k\left[s\left(\alpha_{2}+\varepsilon\right)-\left(\alpha_{2}+\varepsilon\right)\right]^{2}+\pi_{2 \varepsilon} \delta y\right\}$

$L(\varepsilon)$ is the net loss or the net gain to type $\alpha_{2}+\varepsilon$ from imitating type $\alpha_{2}$.

As it is an equilibrium $L(\varepsilon) \leq 0$ for all $\varepsilon>0$.

$L(\varepsilon) \geq \pi_{1} k\left[\left(s\left(\alpha_{2}+\varepsilon\right)-\left(\alpha_{2}+\varepsilon\right)\right)^{2}-\left(s-\alpha_{2}-\varepsilon\right)^{2}\right]+\pi_{1 \varepsilon^{*}}\left(\pi_{2}-\pi_{2 \varepsilon}\right) \delta y \geq$ $\pi_{1} k\left[\left(s\left(\alpha_{2}+\varepsilon\right)-\left(\alpha_{2}+\varepsilon\right)\right)^{2}-\left(s-\alpha_{2}-\varepsilon\right)^{2}\right]+\pi_{1 \varepsilon^{*}} c \delta y$.

$\inf _{\varepsilon>0} \pi_{1} k\left[\left(s\left(\alpha_{2}+\varepsilon\right)-\left(\alpha_{2}+\varepsilon\right)\right)^{2}-\left(s-\alpha_{2}-\varepsilon\right)^{2}\right]=0$, then for $\varepsilon$ small enough $L(\varepsilon)>0$, a contradiction.

Proof of Lemma 3 By contradiction let $s(\widehat{\alpha})=\widehat{\alpha}$ for some $\widehat{\alpha} \in\left(\alpha_{1}, \alpha_{2}\right)$. Let $\varepsilon \geq 0$ and set $\pi_{1 \varepsilon}=\pi_{1}(\widehat{\alpha}+\varepsilon),, \pi_{1}=\pi_{1}, \pi_{2 \varepsilon}=\pi_{2 R}(\widehat{\alpha}+\varepsilon) \pi_{2}=\pi_{20}$. As $s$ is strictly strictly increasing $\pi_{2 \varepsilon}<\pi_{2}$ and $\pi_{1 \varepsilon} \leq \pi_{1}$ for all $\varepsilon>0$. Set $L(\varepsilon)$ be the net loss or the net gain to type $\widehat{\alpha}+\varepsilon$ from imitating type $\widehat{\alpha}$. As it is an equilibrium $L(\varepsilon) \leq 0$ for all $\varepsilon>0$.

$L(\varepsilon)=\pi_{1}\left(y-k \varepsilon^{2}+\pi_{2} \delta y\right)-\pi_{1 \varepsilon}\left\{y-k[s(\widehat{\alpha}+\varepsilon)-(\widehat{\alpha}+\varepsilon)]^{2}+\pi_{2 \varepsilon} \delta y\right\}$

$L(\varepsilon) \geq \pi_{1}\left(y-k \varepsilon^{2}+\pi_{2} \delta y\right)-\pi_{1 \varepsilon}\left\{y+\pi_{2 \varepsilon} \delta y\right\} \geq \pi_{1 \varepsilon^{*}}\left[-k \varepsilon^{2}+\left(\pi_{2}-\pi_{2 \varepsilon}\right) \delta y\right]$, for some fixed $\varepsilon^{*}>0$. $\pi_{1 \varepsilon^{*}}>0$ and $\pi_{2 \varepsilon}=\pi(\widehat{\alpha}+\varepsilon, f(\cdot))$.

Set $B(\varepsilon)=\pi_{1 \varepsilon^{*}}\left[-k \varepsilon^{2}+\left(\pi_{2}-\pi_{2 \varepsilon}\right) \delta y\right]$

$\frac{d B(\varepsilon)}{d \varepsilon}=-\pi_{1 \varepsilon^{*}}\left(2 k \varepsilon+\frac{d \pi_{2 \varepsilon}}{d \varepsilon}\right)$

$\frac{d B(\varepsilon)}{d \varepsilon}_{\mid \varepsilon=0}=-\pi_{1 \varepsilon^{*}}\left(\frac{d \pi(\widehat{\alpha}+\varepsilon, f(\cdot))}{d \varepsilon}_{\mid \varepsilon=0}\right)>0$ from Remark 1. As $B(0)=0$ then $B(\varepsilon)>0$ for $\varepsilon$ small enough. But then type $\widehat{\alpha}+\varepsilon$ could profitably mimic type $\widehat{\alpha}$, for $\varepsilon$ small enough, yielding a contradiction.

By contradiction assume $s(\alpha)>\alpha$. Consider type $\alpha^{\prime}=s(\alpha)>\alpha$. By monotonicity $s\left(\alpha^{\prime}\right)>s(\alpha)$ because agents in $\left[\alpha_{1}, \alpha_{2}\right)$ separate. But then $\alpha^{\prime}$ can profitably imitate $\alpha$ : the probabilities of election at both stages increase (weakly) and she would not pay policy costs.

\subsection{Section 5}

Lemma 5 Let $f$ be a negative $C^{1}$ functions defined on $[0, D] \times B$ where $B$ is a real interval such that. $[0, D] \varsubsetneqq B \subset(-\infty, D]$. Let $f$ be negative on $B$. . Then there exists a solution, defined on $[0, D]$, to the following ordinary differential equation problem: $\left\{\begin{aligned} y^{\prime}(x)(y-x) & =f(x, y(x)) \\ y(D) & =D \\ y(x) & \leq x\end{aligned}\right.$.

If there exists $\delta>0$ such that $f_{y}(x, y) \geq 0(x, y) \in\{(x, y) \in B:\|(x, y)-(D, D)\|<\delta, y<x$,$\} ,$ then the solution is unique.

Proof. The problem does not satisfy the local Lipschitz conditions in a neighborhood of the origin. The existence part of the Proof is by approximation. Let 
$y_{\varepsilon}$ be the solution of the following Cauchy problem

$\left\{\begin{array}{c}y^{\prime}(x)(y(x)-x)=f(x, y(x)) \\ y(D)=D-\varepsilon\end{array}\right.$

.Here the local existence and uniqueness theorem applies. For showing that $y_{\varepsilon}(x)$ can be extended to the interval $[0, D]$ it suffices to show that there exists no $x^{*} \in[0, D)$, such that $\lim _{x \rightarrow x^{*}} y_{\varepsilon}^{\prime}(x)=\infty$. In this case classical extension theorems apply. First observe that if $y_{\varepsilon}$ is defined and $C^{1}$ in the interval $\left(x^{*}, D\right]$ from $y_{\varepsilon}(D)=D-\varepsilon$ and $y_{\varepsilon}^{\prime}(x)\left(y_{\varepsilon}(x)-x\right)<0$ follows $y_{\varepsilon}^{\prime}(x)>0$ and $y_{\varepsilon}(x)<x$ on $\left(x^{*}, D\right]$. If $\lim _{x \rightarrow x^{*}} y_{\varepsilon}^{\prime}(x)=\infty$, then $y_{\varepsilon}(x) \rightarrow x^{*}$ for $x \rightarrow x^{*}$. For small enough $\delta>0 y_{\varepsilon}^{\prime}(x)>2$ on $\left(x^{*}, x^{*}+\delta\right]$. Then let $0<\delta^{\prime}<\delta$. By the intermediate value theorem $y_{\varepsilon}\left(x^{*}+\delta\right)-\left(x^{*}+\delta\right)=y_{\varepsilon}\left(x^{*}+\delta^{\prime}\right)-\left(x^{*}+\delta^{\prime}\right)+y_{\varepsilon}^{\prime}\left(x^{*}+\delta^{\prime \prime}\right)\left(\delta-\delta^{\prime}\right)$ for for some $\delta^{\prime}<\delta^{\prime \prime}<\delta$. but then $y_{\varepsilon}\left(x^{*}+\delta\right)-\left(x^{*}+\delta\right)>y_{\varepsilon}\left(x^{*}+\delta^{\prime}\right)-$ $\left(x^{*}+\delta^{\prime}\right)+2\left(\delta-\delta^{\prime}\right)$. Let $\delta^{\prime} \rightarrow 0$. From the previous observations it follows that the RHS converges to $2 \delta$ while the LHS is independent on $\delta^{\prime}<\delta$. Then $y_{\varepsilon}\left(x^{*}+\delta\right)-\left(x^{*}+\delta\right)>2 \delta>0$, which yields a contradiction. $y_{\varepsilon}(x)$ is $C^{1}$ with respect to $\varepsilon$ on $[0, D)$ (Pontiriaguine (1969), ch. 23). $y_{\varepsilon}(D) \rightarrow D$ for $\varepsilon \rightarrow 0$. Furthermore the family $\left\{y_{\varepsilon}\right\}_{\varepsilon>0}$ is uniformly bounded on each interval $[0, D-\delta], 0<\delta<D$ If $y_{\varepsilon}(x)$ is not converging for $\varepsilon \rightarrow 0$, for some $x \in[0, D)$ Ascoli-Arzelá Theorem. does not apply for some interval $[0, D-\delta]$. It follows that $\sup _{\varepsilon>0} y_{\varepsilon}^{\prime}=\infty$. As above it follows that $y_{\varepsilon}(x)>x$ for some $\varepsilon$ and some $x \in[0, D-\delta]$, a contradiction. So $y_{\varepsilon}$ converges uniformly to some $y$ in each interval $[0, D-\delta]$. each $y_{\varepsilon} y^{\prime}(x)(y(x)-x)=f(x, y(x))$, so $y_{\varepsilon}^{\prime}$ converges uniformly to some continuous $z$. From classical theorems on uniform convergence then satisfies $y^{\prime}=z$. The local existence and uniqueness theorem implies that $y$ is independent on the choice of $\delta . y$ is defined and differentiable on $[0, D)$ and satisfies. $y^{\prime}(x)(y(x)-x)=f(x, y)$ because each $y_{\varepsilon}$ satisfies it. $y(x)<x$ on $[0, D)$ otherwise $y^{\prime}(x) \rightarrow \infty$ for $x \rightarrow x^{*}$, some $x^{*}$ against the uniform convergence of $y_{\varepsilon}^{\prime}$. The existence part is the proved setting $y(D)=\lim _{x \rightarrow D} y(x)=0$. Now assume that $f, g$ satisfy the local properties defined in the claim is locally decreasing as defined in the claim. By contradiction assume that there are two distinct solution, $y$ and $z$ of the problem. The local existence and uniqueness theorem implies that the graphs of the function cross only at $(D, D)$. There is no loss of generality then in assuming that $y(x)<z(x)$ on $[0, D)$. Then $y^{\prime}(x)>z^{\prime}(x)$ for $x \in[D-\delta, D)$ for $0<\delta<D$, small enough. For $x$ next to $D$, we have $z^{\prime}(x)(z(x)-x) \geq y^{\prime}(x)(y(x)-x)=f(x, y(x)) \geq f(x, z(x))$ with at least one strict inequality, a contradiction because $z$ solves the ODE problem.

From Lemma 2 follows that if some types $\left(\alpha^{\prime}, \alpha^{\prime \prime}\right) \subset\left(\alpha_{i}, \alpha_{i+1}\right)$ are in the same policy pool then $\left(\alpha_{i}, \alpha_{i+1}\right)$ is included in the same policy pool. So if $\left(\alpha_{i}, \alpha\right)$ with $\alpha \leq \alpha_{i+1}$ are separating then types in $\left(\alpha_{i}, \alpha_{i+1}\right)$ are all separating.

Lemma 6 In a symmetric MD1 equilibrium

(i) $s(0)=0$

(ii) If types in $\left(\alpha_{i}, \alpha_{i+1}\right)$ are in the same policy pool then agents in $\left(\alpha_{i+1}, \alpha_{i+2}\right)$ separate. 
(iii) If types in $\left(\alpha_{i}, \alpha_{1+1}\right)$ separate then $\alpha_{i+1}=D$

(iv) $s(D)=D$

Proof. For $i=1, \ldots$, Set $s_{i}=\lim _{\alpha \nearrow \alpha_{i}} s(\alpha)$ and set $\overline{s_{i}}=\lim _{\alpha \searrow \alpha_{i}} s(\alpha)$. Set $s_{0}=0, \overline{s_{0}}=s(0) s_{D}=\overline{\lim }_{\alpha \nearrow D} s(\alpha)$ and set $\overline{s_{D}}=s(D) \leq D$..By definition $s_{i}<\overline{s_{i}}$ for $i \overline{=1} 1,2$. and $s_{0} \leq \overline{s_{0}}, s_{D} \leq \overline{s_{D}}$. From equilibrium monotonicity it follows that for all $i=1,2 \ldots, \bar{s} \in\left(\underline{s_{i}}, \overline{s_{i}}\right) T_{1}(\alpha, \beta, s, \pi)<0$ if $\alpha>\alpha_{i}$ and $T_{1}(\alpha, \beta, s, \pi)>0$ for $\alpha<\alpha_{i}$. Then from Condition (2) $\mu\left(\alpha_{i} \mid\right.$ $m(\beta), s)=1$ because $T$ increases as $\alpha$ approaches $\alpha_{i}$. For $s \in\left(\underline{s_{0}}, \overline{s_{0}}\right)$ and $\alpha>0$, $T_{1}(\alpha, \beta, s, \pi)<0$ so $\mu\left(\alpha_{i} \mid m(\beta), s\right)=1$. Finally for all $s \in\left(\underline{s_{D}}, \overline{s_{D}}\right) \cup\left(\overline{s_{D}}, D\right)$, $T_{1}(\alpha, \beta, s, \pi)>0$ then for all $s \in\left(s_{D}, \overline{s_{D}}\right) \cup\left(\overline{s_{D}}, D\right), \mu(D \mid \bar{m}(\beta), s)=1$.

(i) By contradiction let $s(0)>0$. From the result above, for $\varepsilon$ small enough $\mu(0 \mid m(0), \varepsilon)=1$. then $\varepsilon$ can profitably deviate by sending $(m(0), \varepsilon)$.

(ii) Let $s^{*}=s(\alpha)$ for all $\alpha \in\left(\alpha_{i}, \alpha_{i+1}\right)$.. If agents in $\left(\alpha_{i+1}, \alpha_{i+2}\right)$ are pooling then, for $\varepsilon$ small enough $\alpha_{i+1}+\varepsilon$ can profitably deviate by implementing policy $\overline{s_{i}}-\delta$, with $\delta$ small enough. $\mu\left(\alpha_{i+1} \mid m\left(\alpha_{i+1}+\varepsilon\right), \overline{s_{i}}-\delta\right)=1$ The loss in policy term is infinitesimal by continuity of $s$ on $\left(\alpha_{i+1}, \alpha_{i+2}\right)$, the gain in second election probability is bounded below by a positive constant. agent.

(iii) If types in $\left(\alpha_{i}, \alpha_{i+1}\right)$ are separating and types in $\left(\alpha_{i+1}, \alpha_{i+2}\right)$ are pooling then type $\alpha_{i+1}+\varepsilon$ can profitably deviate by sending $\left(m\left(\alpha_{i+1}-\varepsilon\right), \overline{s_{i}}-\delta\right)$. $\mu\left(\alpha_{i+1} \mid\left(m\left(\alpha_{i+1}-\varepsilon\right), \overline{s_{i}}-\delta\right)\right)=1$. For $\delta$ small enough, the loss in policy term is compensated by the gain in election probability. If types in $\left(\alpha, \alpha_{i+1}\right)$ are pooling then, for $\varepsilon$ small enough $\alpha_{i+1}+\varepsilon$ can profitably deviate by implementing policy $\overline{s_{i}}-\delta$, with $\delta$ small enough. $\mu\left(\alpha_{i+1} \mid m\left(\alpha_{i+1}+\varepsilon\right), \overline{s_{i}}-\delta\right)=1$ The loss in policy term is of order $\delta^{2}$, the gain in second election probability is bounded below by a positive constant.

(iv) If $s(D)<D$, for , $\mu(D \mid m(D), D)=1$, because for all $s \in(s(D), D)$ has a maximum between $s$ and $D$ Then $D$ can profitably deviate by implementing policy $D-\varepsilon$, with $\varepsilon<D-s(D)$.

Proof of Proposition 7 It suffices to show that $\alpha^{*}>0$. If $\alpha^{*}=0$, then there would be an MD1 equilibrium with separating policies against Proposition 4

Proof of Theorem 8 We will always assume that whenever beliefs are not imposed by Bayesian consistency or by the monotonic D1 refinement if a candidate announce a policy and implement a different equilibrium policy then the median voter will not confirm her. This is consistent as we allow beliefs to be correlated. Let's consider the different possibilities.

(a) The first one is that $\alpha^{*}=D$ so that the equilibrium is equivalent to an equilibrium in which all types are pooling together at 0 a and and at both stages they are elected with probability $\frac{1}{2}$, and after the the first election all pool on policy 0 . The payoff for type $\alpha$ is $\frac{1}{2}\left[\left(1+\frac{\delta}{2}\right) y-k \alpha^{2}\right]$. This is an MD1 equilibrium if and only if $\frac{1}{2}\left[\left(1+\frac{\delta}{2}\right) y-k D^{2}\right] \geq \frac{1}{2} y$ and $\frac{\delta}{2} y-k D^{2} \geq$ $\delta y \pi_{2}(D)$, otherwise type $D$ could profitably separate by implementing 
policy $D$ (at the campaign and at the policy stage respectively ${ }^{12}$ ) which is as far as

$$
k \leq \min \left\{\frac{y \delta}{2 D^{2}}, y \delta\left(\frac{1}{2}-\pi_{2}(D)\right)\right\}=y \delta\left(\frac{1}{2}-\pi_{2}(D)\right)=k_{0}
$$

where $\pi_{2}(D)$ is the probability a candidate is elected at the second stage if perceived as type $D$ and the other candidate is selected from $F_{L}$, which is with probability $\left[1-G\left(\frac{1}{2} \frac{D^{2}-\int_{0}^{D} \beta^{2} f(\beta) d \beta}{D+\int_{0}^{D} \beta f(\beta) d \beta}\right)\right]<\frac{1}{2}$, because of the symmetry of $G$. For $k<k_{0}$ a (continuous of) pooling equilibrium exists but it does not satisfies the MD1 criterion.

(b) The possibility is that $\alpha^{*}<D$, and all types pool at the first stage. In such a case all types are elected with probability $\frac{1}{2}$ at the first election. At the second stage type $\alpha \in\left[0, \alpha^{*}\right]$ is elected with probability

$$
\pi_{2}\left(\left[0, \alpha^{*}\right]\right)=\left[1-G\left(e\left(\left[0, \alpha^{*}\right], f(\cdot)\right)\right)\right]
$$

where

$$
e\left(\left[0, \alpha^{*}\right], f(\cdot)\right)=\frac{1}{2} \frac{\int_{0}^{\alpha^{*}} \beta^{2} f(\beta) d \beta-F\left(\alpha^{*}\right) \int_{0}^{D} \beta^{2} f(\beta) d \beta}{\int_{0}^{\alpha^{*}} \beta f(\beta) d \beta+F\left(\alpha^{*}\right) \int_{0}^{D} \beta f(\beta) d \beta}
$$

is the decisive voter when the types in $\left[0, \alpha^{*}\right]$ are pooling and matched to a challenger selected from $F_{L}$ on $[-D, 0]$. Elementary analysis shows that $-D<e\left(\left[0, \alpha^{*}\right], f(\cdot)\right)<D$ for $\alpha^{*}>0$ and $e([0, \alpha], f(\cdot))$ it is strictly increasing. Furthermore $\lim _{\alpha^{*} \rightarrow 0^{+}} e\left(\left[0, \alpha^{*}\right], f(\cdot)\right)=-\frac{1}{2} \frac{\int_{0}^{D} \beta^{2} f(\beta) d \beta}{\int_{0}^{D} \beta f(\beta) d \beta} \in$ $[-D, 0] . \lim _{\alpha^{*} \rightarrow D^{-}} e\left(\left[0, \alpha^{*}\right], f(\cdot)\right)=0$. So $\pi_{2}(\alpha)$ is strictly decreasing and differentiable in $\alpha$.

On the other side, a type $\alpha \in\left(\alpha^{*}, D\right]$ is elected at the second stage with probability

$$
\pi_{2}(\alpha)=1-G(e(\alpha, f(\cdot)))
$$

where

$$
e(\alpha, f(\cdot))=\frac{1}{2} \frac{\alpha^{2}-\int_{0}^{D} \beta^{2} f(\beta) d \beta}{\alpha+\int_{0}^{D} \beta f(\beta) d \beta}
$$

We have $-D<e\left(\left[0, \alpha^{*}\right], f(\cdot)\right)<e(\alpha, f(\cdot))<D$. So $\pi_{2}(\alpha)<\pi_{2}\left(\left[0, \alpha^{*}\right]\right)$. for $\alpha>\alpha^{*}$. $e(\alpha, f(\cdot))$ is strictly increasing on $\left(\alpha^{*}, D\right] . \lim _{\alpha^{*} \rightarrow D^{-}} e(\alpha, f(\cdot))=$ $\frac{1}{2} \frac{D^{2}-\int_{0}^{D} \beta^{2} f(\beta) d \beta}{D+\int_{0}^{D} \beta f(\beta) d \beta} \in(0, D)$ because So $\pi_{2}(\alpha)$ is strictly decreasing and continuously differentiable in $\alpha^{*}$.

If $s(\alpha)$ is separating on $\left(\alpha^{*}, D\right]$ the it must satisfy

$$
\left.2 k s^{\prime}(\alpha)\right)(s(\alpha)-\alpha)=\pi_{2}^{\prime}(\alpha) \delta y
$$

\footnotetext{
${ }^{12}$ See the proof of Proposition 7 above.
} 
with the final condition $s(D)=D$. Furthermore $\alpha^{*}=\alpha_{1}(k)>0$ must be indifferent between separating and pooling, which is it must satisfy

$$
\left(\frac{1}{2}+\delta \pi_{2}\left(\left[0, \alpha^{*}\right]\right)\right) y-k \alpha^{* 2}=\left(\frac{1}{2}+\delta \pi_{2}\left(\alpha^{*}\right)\right) y-k\left(s\left(\alpha^{*}\right)-\alpha^{*}\right)^{2}
$$

Set $H(\alpha)=\left(\frac{1}{2}+\delta \pi_{2}([0, \alpha])\right) y-k \alpha^{2}-\left(\frac{1}{2}+\pi_{2}(\alpha) \delta\right) y+k(s(\alpha)-\alpha)^{2}$. $s(0)<0$. So $H(0)>0$. $H(D)=\left[\pi_{2}([0, D])-\pi_{2}(D)\right] \delta y-k D^{2}=$ $\left[\frac{1}{2}-\pi_{2}(D)\right] \delta y-k D^{2} \leq 0$ if $k \geq k_{0} . H^{\prime}(\alpha)<0=\frac{d \pi_{2}([0, \alpha])}{d \alpha} \delta y-2 k s(\alpha)^{13}<$ 0 . There exists a unique such $\alpha_{1}(k)>0$. It is easily seen that $s\left(\alpha_{1}(k)\right)>0$ because if $s(\alpha)=0$ then $H(\alpha)>0$.

Through implicit differentiation

so,

$$
\frac{d H\left(\alpha_{1}(k)\right)}{d k}=H_{\alpha}\left(\alpha_{1}(k)\right) \frac{d \alpha_{1}(k)}{d k}+H_{k}\left(\alpha_{1}(k)\right)=0
$$

$$
\frac{d \alpha_{1}(k)}{d k}=\frac{-H_{k}\left(\alpha_{1}(k)\right)}{H_{\alpha}\left(\alpha_{1}(k)\right)}=-\frac{s^{2}\left(\alpha_{1}(k)\right)-2 \alpha_{1}(k) s\left(\alpha_{1}(k)\right)}{\frac{d \pi_{2}\left(\left[0, \alpha_{1}(k)\right]\right)}{d \alpha} \delta y-2 k s\left(\alpha_{1}(k)\right)}<0
$$

Then $\alpha_{1}(k)$ is strictly decreasing in $k$.

From $H\left(\alpha_{1}(k)\right)=0$ follows $\alpha_{1}(k) \rightarrow 0$ as $k \rightarrow \infty$.

(3) Another possibility is that there are two campaign pools $\left[0, \alpha^{*}\right)$ and $\left(\alpha^{*}, D\right]$, with the second separating in policies. $\left[0, \alpha^{*}\right)$ types' election probabilities are

$$
\bar{\pi}_{1}\left(\left[0, \alpha^{*}\right]\right)=\frac{1}{2} F\left(\alpha^{*}\right)+\left(1-F\left(\alpha^{*}\right)\right)\left[1-G\left(e\left(\left[0, \alpha^{*}\right),\left(\alpha^{*}, D\right]\right)\right)\right]
$$

and $\pi_{2}\left(\left[0, \alpha^{*}\right)\right)$, respectively, where $\left(e\left(\left[0, \alpha^{*}\right),\left(\alpha^{*}, D\right]\right)\right)$ is the decisive median voter of pool $\left[0, \alpha^{*}\right)$ against pool $\left(\alpha^{*}, D\right]$ : her location is

$$
\begin{aligned}
& \frac{1}{2} \frac{\left(1-F\left(\alpha^{*}\right)\right) \int_{0}^{\alpha^{*}} \beta^{2} f(\beta) d \beta-F\left(\alpha^{*}\right) \int_{\alpha^{*}}^{D} \beta^{2} f(\beta) d \beta}{\left(1-F\left(\alpha^{*}\right)\right) \int_{0}^{\alpha^{*}} \beta^{2} f(\beta) d \beta+F\left(\alpha^{*}\right) \int_{\alpha^{*}}^{D} \beta^{2} f(\beta) d \beta} \\
& \frac{1}{2} \frac{\int_{0}^{\alpha^{*}} \beta^{2} f(\beta) d \beta-F\left(\alpha^{*}\right) \int_{0}^{D} \beta^{2} f(\beta) d \beta}{\int_{0}^{\alpha^{*}} \beta^{2} f(\beta) d \beta+F\left(\alpha^{*}\right)\left[\int_{\alpha^{*}}^{D} \beta^{2} f(\beta) d \beta-\int_{0}^{\alpha^{*}} \beta^{2} f(\beta) d \beta\right]}
\end{aligned}
$$

Observe that $G\left(e\left(\left[0, \alpha^{*}\right),\left(\alpha^{*}, D\right]\right)\right) \leq \frac{1}{2}$, because $e\left(\left[0, \alpha^{*}\right),\left(\alpha^{*}, D\right]\right) \leq 0$. $\left(\alpha^{*}, D\right]$ 's election probabilities are

$$
\bar{\pi}_{1}\left(\left(\alpha^{*}, D\right]\right)=\left(F\left(\alpha^{*}\right)\right)\left(1-G\left(e\left(\left(\alpha^{*}, D\right],\left[0, \alpha^{*}\right)\right)\right)\right)+\frac{1}{2}\left(1-F\left(\alpha^{*}\right)\right)
$$

and $\pi_{2}(\alpha)$, respectively, where

$$
e\left(\left(\alpha^{*}, D\right],\left[0, \alpha^{*}\right)\right)=\frac{1}{2} \frac{F\left(\alpha^{*}\right) \int_{\alpha^{*}}^{D} \beta^{2} f(\beta) d \beta-\left(1-F\left(\alpha^{*}\right)\right) \int_{0}^{\alpha^{*}} \beta^{2} f(\beta) d \beta}{F\left(\alpha^{*}\right) \int_{\alpha^{*}}^{D} \beta^{2} f(\beta) d \beta+\left(1-F\left(\alpha^{*}\right)\right) \int_{0}^{\alpha^{*}} \beta^{2} f(\beta) d \beta}
$$

\footnotetext{
${ }^{13}$ Because $s$ solves the differential equation.
} 
From the symmetry of the distribution $G, G\left(e\left(\left[0, \alpha^{*}\right),\left(\alpha^{*}, D\right]\right)\right)=1-$ $G\left(e\left(\left(\alpha^{*}, D\right],\left[0, \alpha^{*}\right)\right)\right)=1-G \leq \frac{1}{2}$. so

$$
\bar{\pi}_{1}\left(\left(\alpha^{*}, D\right]\right)=\left(F\left(\alpha^{*}\right)\right)\left(G\left(e\left(\left[0, \alpha^{*}\right),\left(\alpha^{*}, D\right]\right)\right)\right)+\frac{1}{2}\left(1-F\left(\alpha^{*}\right)\right)
$$

and $\bar{\pi}_{1}\left(\left[0, \alpha^{*}\right]\right)=\bar{\pi}_{1}\left(\left(\alpha^{*}, D\right]\right)+\frac{1}{2}-G\left(e\left(\left(\alpha^{*}, D\right],\left[0, \alpha^{*}\right)\right)\right) \geq \bar{\pi}_{1}\left(\left(\alpha^{*}, D\right]\right)$. As above, on $\left(\alpha^{*}, D\right] s$ must satisfy

$$
\left.2 k s^{\prime}(\alpha)\right)(s(\alpha)-\alpha)=\pi_{2}^{\prime}(\alpha) \delta y
$$

and $\alpha^{*}$ must satisfy

$\bar{\pi}_{1}\left[0, \alpha^{*}\right]\left[1+\pi_{2}\left(\left[0, \alpha^{*}\right]\right) \delta y-k \alpha^{* 2}\right]=$

$\bar{\pi}_{1}\left(\left(\alpha^{*}, D\right]\right)\left[1+\pi_{2}\left(\alpha^{*}\right) \delta y-k\left(s\left(\alpha^{*}\right)-\alpha^{*}\right)^{2}\right]$

Set $H(\alpha, k)=\left\{\left(\bar{\pi}_{1}\left(\left[0, \alpha^{*}\right]\right)+\delta \pi_{2}\left(\left[0, \alpha^{*}\right]\right)\right) y-k \alpha^{* 2}\right\}-$ $-\left\{\left(\bar{\pi}_{1}\left(\left(\alpha^{*}, D\right]\right)+\pi_{2}\left(\alpha^{*}\right) \delta\right) y-k\left(s\left(\alpha^{*}\right)-\alpha^{*}\right)^{2}\right\}$

As above it can be shown, that a unique solution to $H\left(\alpha_{2}(k), k\right)=0$ exists if and only if $H(D)>0$ which is if and only if $k \geq k_{1}^{*}>k_{0}$ where $H\left(D, k_{1}^{*}\right)=0 . \alpha_{2}(k)$ is strictly decreasing and $\alpha_{2}(k) \rightarrow 0$ as $k \rightarrow \infty$.

It must be checked that type $D$ does not want to imitate type $\alpha_{2}(k)$ in the campaign and then implement $D$. which is

$\bar{\pi}_{1}\left[0, \alpha_{2}(k)\right] y \leq \bar{\pi}_{1}\left(\left(\alpha_{2}(k), D\right]\right)\left[\left(1+\pi_{2}(D)\right) \delta y\right]$, or, equivalently $\frac{1}{2}-G-\bar{\pi}_{1}\left(\alpha_{2}(k), D\right) \pi_{2}(D) \delta \leq$

0 but $\bar{\pi}_{1}\left(\alpha_{2}(k), D\right)=-F\left(\alpha_{2}(k)\right)\left(\frac{1}{2}-G\right)+\frac{1}{2}$ Then he condition is $\left(\frac{1}{2}-G\right)\left(1+F\left(\alpha_{2}(k)\right) \pi_{2}(D) \delta\right) \leq$ $\frac{\pi_{2}(D) \delta}{2}$. Consider the function $R(\alpha)=\left(\frac{1}{2}-G(e([0, \alpha),(\alpha, D]))\right)\left(1+F(\alpha) \pi_{2}(D) \delta\right)-$

$\frac{\pi_{2}(D) \delta}{2} . R(0)=-\frac{\pi_{2}(D) \delta}{2}<0, R^{\prime}>0$. As $\alpha_{2}(k) \searrow 0$ as $k \rightarrow \infty$, there exists a unique $k^{*}>0$ such that this kind of equilibrium exists only for $k \geq k^{*}$. Set $k_{1}=\max \left\{k^{*}, k_{1}^{*}\right\}$.

(d) The last possible case is that agents in $\left[0, \alpha^{*}\right]$ pool in campaign and policy, and agents in $(\alpha, D]$ separate in campaign and in message. Then the probability of first stage election election of types in in $\left[0, \alpha^{*}\right]$ is

$$
\pi_{1}\left(\left[0, \alpha^{*}\right]\right)=\frac{1}{2} F\left(\alpha^{*}\right)+\int_{\alpha^{*}}^{D}\left[1-G\left(e\left(\left[0, \alpha^{*}\right], \beta\right)\right)\right] f(\beta) d \beta
$$

where, for all $\alpha>\alpha^{*}$

$$
e\left(\left[0, \alpha^{*}\right], \alpha\right)=\frac{1}{2} \frac{\int_{0}^{\alpha^{*}} \beta^{2} f(\beta) d \beta-F\left(\alpha^{*}\right) \alpha^{2}}{\int_{0}^{\alpha^{*}} \beta^{2} f(\beta) d \beta+F\left(\alpha^{*}\right) \alpha}
$$

$e\left(\left[0, \alpha^{*}\right], \alpha\right)<0$ as $\alpha>\alpha^{*}$. So $G\left(e\left(\left[0, \alpha^{*}\right], \alpha\right)\right)<\frac{1}{2}$.

If $\alpha^{*}<\alpha<D$ then is separating in campaign $D$ is elected with probability

$$
\pi_{1}(\alpha)=F\left(\alpha^{*}\right)\left(1-G\left(e\left(\alpha,\left[0, \alpha^{*}\right]\right)\right)\right)+\frac{1}{2} \int_{\alpha^{*}}^{D}\left[1-G\left(\frac{\beta+\alpha}{2}\right)\right] f(\beta) d \beta
$$


where

$$
e\left(\alpha,\left[0, \alpha^{*}\right]\right)=\frac{1}{2} \frac{F\left(\alpha^{*}\right) \alpha^{2}-\int_{0}^{\alpha^{*}} \beta^{2} f(\beta) d \beta}{\int_{0}^{\alpha^{*}} \beta^{2} f(\beta) d \beta+F\left(\alpha^{*}\right) \alpha}=-e\left(\left[0, \alpha^{*}\right], \alpha\right)
$$

So $G\left(e\left(\alpha,\left[0, \alpha^{*}\right]\right)\right)=\frac{1}{2}+G\left(e\left(\left[0, \alpha^{*}\right],\right)\right)$

We can write

$$
\begin{aligned}
\pi_{1}\left(\left[0, \alpha^{*}\right]\right)= & \frac{1}{2} F\left(\alpha^{*}\right)+\int_{\alpha^{*}}^{D}\left[\frac{3}{2}-G\left(\beta, e\left(\left[0, \alpha^{*}\right]\right)\right)\right] f(\beta) d \beta= \\
& \frac{3}{2}-F\left(\alpha^{*}\right)-\int_{\alpha^{*}}^{D}\left[G\left(\beta, e\left(\left[0, \alpha^{*}\right]\right)\right)\right] f(\beta) d \beta
\end{aligned}
$$

Now $s$ must satisfy the differential equation

$\pi_{1}^{\prime}(\alpha)\left[y-k(s(\alpha)-\alpha)^{2}+\pi_{2}(\alpha) \delta y\right]+$

$\left.\left.\pi_{1}(\alpha)\right)\left[-2 k s^{\prime}(\alpha)\right)(s(\alpha)-\alpha)+\pi_{2}^{\prime}(\alpha) \delta y\right]=0$

And $\alpha^{*}=\alpha_{3}(k)$ has to satisfy $\pi_{1}\left(\left[0, \alpha^{*}\right]\right)\left[1+\pi_{2}\left(\left[0, \alpha^{*}\right]\right) \delta y-k \alpha^{* 2}\right]=$ $\pi_{1}\left(\alpha^{*}\right)\left[1+\pi_{2}\left(\alpha^{*}\right) \delta y-k\left(s\left(\alpha^{*}\right)-\alpha^{*}\right)^{2}\right]$

Exactly as above one can prove the existence and uniqueness of $\alpha_{3}(k)$ and of $k_{2}$ such that the strategies are an equilibrium iff $k \geq k_{2}$. 